\title{
Effect of topiramate and zonisamide on fMRI cognitive networks
}

OPEN

Britta Wandschneider,

$\mathrm{PhD}$

Jane Burdett, DCRR

Lucy Townsend

Andrea Hill, DcR (R)

Pamela J. Thompson,

$\mathrm{PhD}$

John S. Duncan,

FMedSci, FRCP

Matthias J. Koepp, PhD

Correspondence to

Dr. Koepp:

m.koepp@ucl.ac.uk
Supplemental data at Neurology.org

\section{ABSTRACT}

Objective: To investigate the effects of topiramate (TPM), zonisamide (ZNS), and levetiracetam (LEV) on cognitive network activations in patients with focal epilepsy using an fMRI language task.

Methods: In a retrospective, cross-sectional study, we identified patients from our clinical database of verbal fluency fMRI studies who were treated with either TPM $(n=32)$ or ZNS $(n=$ 51). We matched 62 patients for clinical measures who took LEV but not TPM or ZNS. We entered antiepileptic comedications as nuisance variables and compared out-of-scanner psychometric measures for verbal fluency and working memory between groups.

Results: Out-of-scanner psychometric data showed overall poorer performance for TPM compared to ZNS and LEV and poorer working memory performance in ZNS-treated patients compared to LEV-treated patients. We found common fMRI effects in patients taking ZNS and TPM, with decreased activations in cognitive frontal and parietal lobe networks compared to those taking LEV. Impaired deactivation was seen only with TPM.

Conclusions: Our findings suggest that TPM and ZNS are associated with similar dysfunctions of frontal and parietal cognitive networks, which are associated with impaired performance. TPM is also associated with impaired attenuation of language-associated deactivation. These studies imply medication-specific effects on the functional neuroanatomy of language and working memory networks.

Classification of evidence: This study provides Class III evidence that in patients with focal epilepsy, TPM and ZNS compared to LEV lead to disruption of language and working memory networks. Neurology ${ }^{\circledR}$ 2017;88:1165-1171

\section{GLOSSARY}

AED = antiepileptic drug; DMN = default mode network; IFG = inferior frontal gyri; LEV = levetiracetam; MFG = middle frontal gyri; TPM = topiramate; ZNS = zonisamide.

Among newer antiepileptic drugs (AEDs), highest rates of cognitive impairment have been reported for topiramate (TPM; Topamax; Janssen-Cilag, Neuss, Germany) and zonisamide (ZNS; Zonegran; EISAI, Tokyo, Japan), leading to early treatment discontinuation. ${ }^{1}$ For TPM, cognitive dysfunction is specifically characterized by impairment of expressive language and working memory. ZNS treatment leads to similar, less pronounced impairment. ${ }^{2,3}$ The mechanisms of these specific impairment patterns are poorly understood. Five cognitive fMRI studies used language tasks in 2 healthy participants, 5 to 16 patients with epilepsy, and 10 patients with migraine after a single dose or on steady-state TPM treatment. Taken together, the following patterns of dysfunctional activation emerge: decreased activation in task-positive regions, i.e., dominant inferior and middle frontal gyri (IFG and MFG), ${ }^{4-6}$ and failure to deactivate tasknegative regions, including the default mode network (DMN). $5,7,8$

From the Department of Clinical and Experimental Epilepsy (B.W., J.B., A.H., P.J.T., J.S.D., M.J.K.), UCL Institute of Neurology, London; and MRI Unit (B.W., J.B., L.T., A.H., P.J.T., J.S.D., M.J.K.), Epilepsy Society, Chalfont St. Peter, UK.

Go to Neurology.org for full disclosures. Funding information and disclosures deemed relevant by the authors, if any, are provided at the end of the article. The Article Processing Charge was paid by the authors.

This is an open access article distributed under the terms of the Creative Commons Attribution-NonCommercial-NoDerivatives License 4.0 (CC BY-NC-ND), which permits downloading and sharing the work provided it is properly cited. The work cannot be changed in any way or used commercially without permission from the journal. 
Table $1 \quad$ Clinical measures

\begin{tabular}{|c|c|c|c|c|c|}
\hline & On LEV ( $n=62)$ & On ZNS $(n=51)$ & On TPM $(n=32)$ & $d f$ & p Value \\
\hline Age, median (IQR), y & $34(26-42)$ & $35(29-42)$ & $35(28-47)$ & 2 & 0.804 \\
\hline Male sex, $n$ & 27 & 28 & 14 & 2 & 0.43 \\
\hline Handedness (right/left/ambidextrous), n & $55 / 5 / 1$ & $42 / 7 / 1$ & $25 / 6 / 1$ & 4 & 0.631 \\
\hline Age at onset, median (IQR), y & $14(7-21)$ & $13(6-19)$ & $11(6-18)$ & 2 & 0.610 \\
\hline Duration, median (IQR), y & $18(12-26)$ & $22(13-31)$ & $23(10-30)$ & 2 & 0.362 \\
\hline Ictal EEG available, $\mathrm{n}$ & 52 & 45 & 28 & 2 & 0.64 \\
\hline Lesion, one/bilateral/left/right, $n$ & $25 / 2 / 19 / 16$ & $26 / 0 / 15 / 10$ & $15 / 3 / 6 / 8$ & 6 & 0.284 \\
\hline Dose, median (IQR), mg & $2,000(1,250-3,000)$ & $225(150-400)$ & $312.5(200-500)$ & 2 & N/A \\
\hline AEDs, median (IQR), $n$ & $2(2-3)$ & $2(2-3)$ & $3(2-3)$ & 2 & 0.245 \\
\hline Patients on monotherapy vs polytherapy & $5 / 57$ & $4 / 47$ & $4 / 28$ & 2 & 0.730 \\
\hline Laterality index, median (IQR) & $0.75(0.53-0.85)$ & $0.77(0.55-0.92)$ & $0.72(0.23-0.84)$ & 2 & 0.391 \\
\hline Scanned on upgraded scanner, $\mathrm{n}$ & 26 & 27 & 8 & 2 & 0.041 \\
\hline
\end{tabular}

Abbreviations: AED = antiepileptic drug; IQR = interquartile range between pair of quartiles; LEV = levetiracetam; TPM = topiramate; ZNS = zonisamide.

Pearson $\chi^{2}$ was used for dichotomous variables, and Kruskal-Wallis test was used for all other variables $(p<0.05)$.

Only one study investigated potential pathomechanisms of cognitive impairment due to ZNS, describing decreased currentsource density of high beta frequency in regions relevant to language and working memory during a verbal fluency task. ${ }^{9}$ All of the above studies are hampered by small sample sizes.

We therefore aimed to investigate in a larger group of patients than previous studies how TPM and ZNS alter fMRI activation patterns to identify patients at risk of developing cognitive side effects. On the basis of previously observed effects of levetiracetam (LEV; Keppra; UCB, Brussels, Belgium) leading to normalization of cognitive network activation in focal epilepsy, ${ }^{10}$ we selected patients on LEV as a comparison group to control for disease-related effects.

METHODS Participants. In this cross-sectional study, we chose patients retrospectively from a clinical database of patients with drug-refractory epilepsy who had undergone clinical language fMRI scans at the UCL Chalfont Centre for Epilepsy (UK) between March 2010 and October 2015 as part of their presurgical evaluation. All patients were adults and seen at the adult epilepsy clinics of the National Hospital for Neurology and Neurosurgery and Chalfont Centre for Epilepsy. We included patients who were taking 1 of the following 3 AEDs: TPM, ZNS, or LEV, either alone or with other AEDs as comedications.

As requirements for patients' testability with the language fMRI paradigm, all patients had to be literate, proficient in the English language, and cognitively able to understand the simple task instructions (see paradigm description below). Our standard clinical language fMRI paradigm is conducted covertly; hence, we could not control for task compliance. For this reason, we excluded all patients without activations of language-relevant regions (IFG and MFG) from the analysis. We also excluded patients with data acquired postoperatively and those with large lesions or tumors $(>2 \mathrm{~cm})$ to avoid problems with imaging normalization and further statistical analysis. Seventy-eight patients on LEV, 51 on ZNS, and 32 on TPM were eligible. To create more balanced group sizes and to ensure that the TPM and ZNS groups were comparable in demographics and clinical characteristics to the LEV group, we used propensity score matching. We started with the ZNS and TPM groups and in each group looked for propensity-matched LEV patients for the variables of age at scan, age at disease onset, sex, language laterality index, total number of medications, and lesion laterality using propensity scores in SPSS version 21.0. We included 51 patients on ZNS, 32 on TPM, and 62 on LEV in the final analysis. All patients had refractory focal epilepsy, and ictal EEG data were available in $86 \%$ of cases. Table 1 and table e- 1 at Neurology.org provide further clinical details.

Primary research question and classification of level of evidence. The primary research goal was to assess the effect of TPM and ZNS on language task fMRI activation and deactivation in patients with focal epilepsy (level of evidence: Class III).

MRI data acquisition and fMRI paradigm. Gradient echoplanar images were acquired for blood oxygen level-dependent contrast on a 3T General Electric Excite HDx scanner (General Electric, Milwaukee, WI).

Each volume comprised 50 contiguous oblique axial slices, providing full brain coverage, with 2.5 -mm slice thickness, $64 \times 64$ matrix, 24-cm field of view, $3.75 \times 3.75$-mm in-plane resolution, 25-millisecond echo time, and 2.5-second repetition time.

Patients performed a covert verbal fluency task lasting for 5.5 minutes. During the paradigm, 30-second blocks of task alternated with 30-second blocks of cross-hair fixation as a control condition. Patients were instructed to covertly generate words starting with a visually presented letter (A, D, E, S, W).

fMRI data analysis. $\mathrm{fMRI}$ analysis was performed with Statistical Parametric Mapping-8, version 4290 (http://www.fil.ion. ucl.ac.uk/spm/). Preprocessing included realignment, spatial normalization to a template in Montreal Neurological Institute 


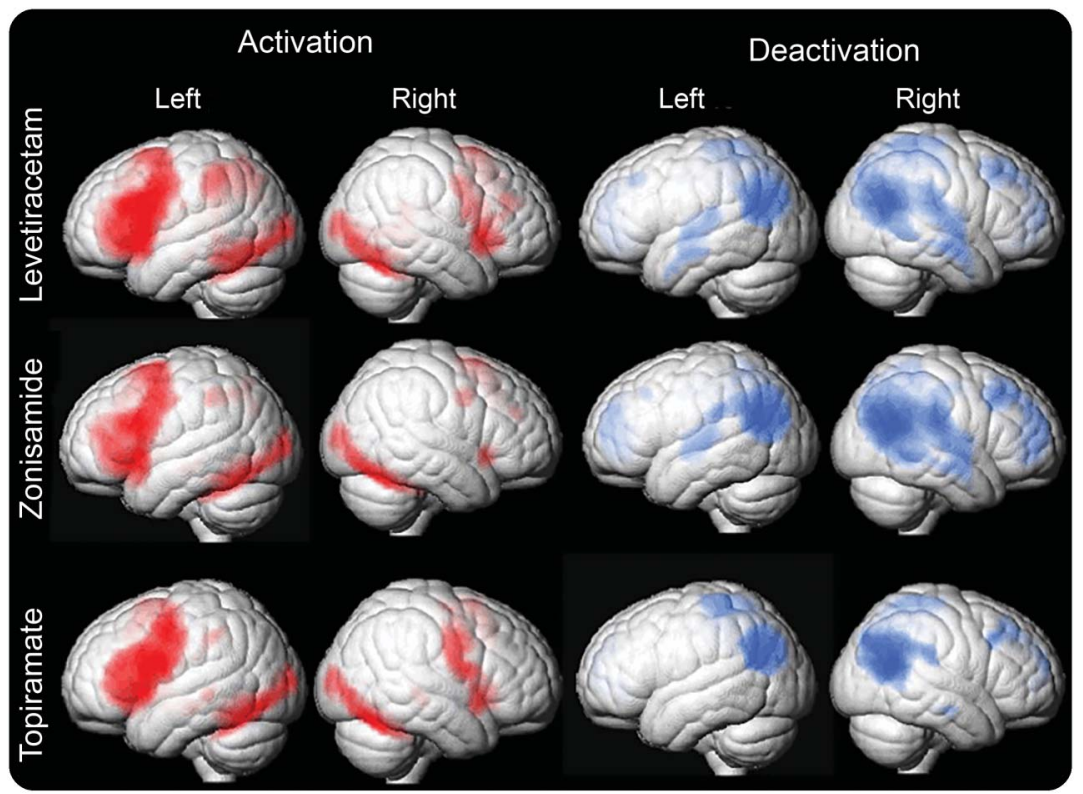

One-sample $t$ tests of $\mathrm{fMRI}$ activation and deactivation maps for the 3 different patient groups on levetiracetam, zonisamide, and topiramate are demonstrated on a surface-rendered brain template. Task-relevant regions (red) include bilateral inferior and middle frontal gyrus (left > right), bilateral supplementary motor area, and the left dorsolateral parietal region. Areas of task-related deactivations (blue) include the bilateral precuneus, posterior cingulate, angular gyrus, and medial prefrontal and lateral temporal cortex. $p<0.005,20$-voxel threshold extent.

space, resampling to isotropic $3 \times 3 \times 3$ voxels, and smoothing with a gaussian kernel of 8 -mm full-width at half-maximum.

We performed the statistical fMRI analyses at the first level, i.e., single subject, and then at the group level. At the first level, the task was modeled by convolving the vector of block onsets with a canonical hemodynamic response function to create regressors of interest. Movement parameters were included as confounds. Contrast images for each participant were created for task-relevant activation and deactivation.

At the second level, we first explored activation and deactivation maps during the verbal fluency task in each patient group using one-sample $t$ tests (figure 1, figure e-1). For group comparisons, second-level analysis was conducted by entering the activation contrast of each patient into a full factorial design with group (LEV, ZNS, TPM) as a factor. All other AEDs were entered as regressors of no interest. Because the scanner was upgraded in 2013, this was entered as an additional covariate of no interest (scanned on upgraded scanner yes/no). An exploratory statistical threshold was set at $p<0.005$ uncorrected with a 20 -voxel minimum cluster size extent threshold. ${ }^{11}$ To be able to disentangle whether group differences were related to activation or deactivation, we masked the results with a binarized average task activation map of the controls and subsequently with the binarized deactivation map to include the contrast-relevant brain areas.

Interpretation of the results at the first level and group level was not blinded because resultant maps represent $t$ maps at a predefined statistical threshold. We anatomically objectified peak activations from group comparisons with coordinates in Montreal Neurological Institute space.

Laterality index. To control for differences in language laterality, we matched the groups for laterality indexes. These were calculated with the bootstrap method in the Statistical Parametric Mapping-8 LI toolbox ${ }^{12}$ for the verbal fluency activation contrast for each participant in the IFG and MFG.

Cognitive measures. In those patients who had standard clinical psychometric testing at the time of the scan, we carried out subgroup analyses of performances. We included tests measuring cognitive domains reported to be affected by TPM and ZNS: letter and category fluency, the Wechsler Adult Intelligence Scale Digit Span

\begin{tabular}{|lllllll}
\hline Table $2 \quad$ Cognitive performance & & & & & \\
& LEV & ZNS & TPM & df & $p$ Value \\
WAIS Digit Span, median (IQR) & $9(7.5-11)(n=42)$ & $7(6-9)(n=36)$ & $5(4.75-7)(n=20)$ & 2 & $<0.001$ \\
Letter fluency, median (IQR) & $14.5(10.25-17.75)(n=38)$ & $12(8-15)(n=35)$ & $10(8-11.5)(n=20)$ & 2 & 0.003 \\
Categorical fluency, median (IQR) & $18.5(15-25.75)(n=39)$ & $17(15-22)(n=35)$ & $12(11-16)(n=20)$ & 2 & $<0.001$ \\
Naming (GNT), median (IQR) & $17(13.45-21.25)(n=34)$ & $17(14-20)(n=28)$ & $14(10.5-17)(n=15)$ & 2 & 0.391 \\
\hline
\end{tabular}

Abbreviations: GNT = Grades Naming Test; IQR = interquartile range between pair of quartiles; LEV = levetiracetam; TPM = topiramate; WAIS = Wechsler Adult Intelligence Scale; ZNS = zonisamide.

Kruskal-Wallis test, $p<0.05$.

Scaled scores are presented for digit span; raw scores are presented for all other cognitive measures. 
measure of short term and working memory, and the Graded Naming Test measure of expressive language function. ${ }^{13}$

Statistical analysis of clinical and behavioral data was conducted with SPSS version 21.0 (IBM, Armonk, NY). We applied $\chi^{2}$ tests to categorical data and the Kruskal-Wallis or MannWhitney $U$ test to all other measures. The statistical significant threshold was set at $p<0.05$.

Standard protocol approvals, registrations, and patient consents. This study was approved by the Joint Ethics Committee of the National Hospital for Neurology and Neurosurgery and University College London Institute of Neurology. The Research Ethics Committee classified this work as evaluation of clinical services, i.e., routine language $\mathrm{PMRI}$; therefore, individual consent from patients was not needed.

RESULTS Cognitive performance. There were significant group differences in cognitive test performance with the exception of the Graded Naming Test (table 2).

Post hoc group comparisons revealed that for the digit span task, patients on LEV performed better than both those on ZNS and TPM (LEV vs ZNS: $U=$ 451000, $p=0.002$; LEV vs TPM: $U=120000, p<$ 0.001 , Mann-Whitney $U$ test), and those on ZNS performed better than patients on TPM (LEV vs ZNS: $U=205000, p=0.007)$. TPM-treated patients performed less well than both LEV- and ZNS-treated patients on both fluency measures (LEV vs TPM: letter fluency $U=177000, p=0.001$, category fluency $U=149500, p<0.001 ; Z N S$ vs TPM: letter fluency $U=237000, p=0.047$; category fluency $U=$ 145500, $p<0.001)$. There was no statistical difference between LEV- and ZNS-treated patients (letter fluency $U=505500 ; p=0.077$; category fluency $U=604500, p=0.397$ ).

fMRI results. As demonstrated by one-sample $t$ tests of task-relevant activations and deactivations, each AED group activated frontal language areas, including the IFG, MFG, bilateral supplementary motor areas, and left lateral parietal region, and deactivated areas of the $\mathrm{DMN}$, i.e., bilateral precuneus, posterior cingulate, angular gyrus, and medial prefrontal and lateral temporal cortices (figure 1, figure e-1).

Comparison of TPM, ZNS, and LEV groups. Patients receiving TPM showed reduced activation in the left MFG and left dorsal parietal region compared to those taking LEV (figure 2A, figure e-2). Similarly, those on ZNS had reduced activation in the bilateral MFG and IFG and the left dorsal parietal region compared to patients on LEV (figure 2B, figure e-2). Compared to LEV patients, TPM- but not ZNS-treated patients showed less task-related deactivation in the temporal regions and the rolandic opercula bilaterally, as well as the right inferior parietal lobule and supramarginal gyrus (figure $2 \mathrm{C}$, figure e-2).

Comparing TPM- and ZNS-treated patients directly revealed greater activation in the temporal
Figure 2 Group differences in $\mathrm{fMR}$ activation maps during the verbal fluency task

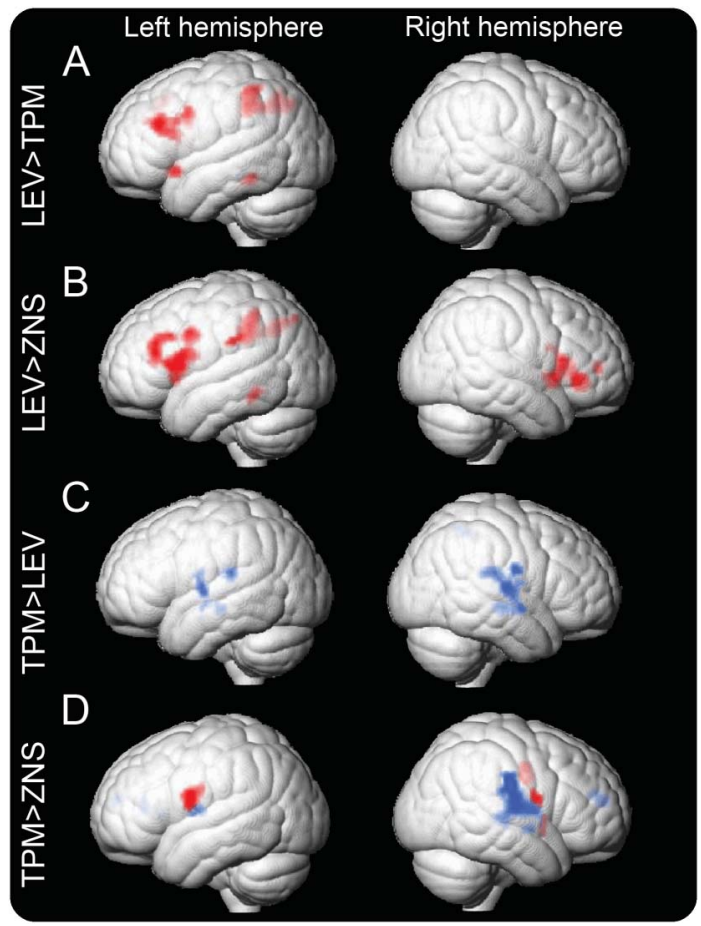

Significant group differences between patients on levetiracetam (LEV), topiramate (TPM), and zonisamide (ZNS) are demonstrated. Patients on TPM and ZNS have less activation in frontal and parietal cognitive networks than patients on LEV. In patients on TPM, activation is reduced in the left middle frontal gyrus (MFG) and left dorsal parietal region (A). In patients on ZNS, activation is reduced in the left MFG and bilateral inferior frontal gyrus (IFG), as well as the left dorsal parietal region (B). In terms of task-relevant deactivation networks, bilateral lateral temporal regions and rolandic opercula and the right inferior parietal lobule and supramarginal gyrus are less deactivated (blue) in patients on TPM compared to those on LEV (C). Compared to ZNS, TPM shows increased activation in the IFG, insular cortex, and rolandic operculum on the left and the insular cortex, inferior parietal lobule, supramarginal gyrus, superior temporal gyrus, and rolandic operculum on the right. Differences in the left are due mainly to increased activation of task-relevant regions as shown in red (inclusively masked with LEV activation maps); on the right, activated regions lie mainly within task-negative areas, i.e., are due to impaired deactivation as shown in blue (inclusively masked with LEV and ZNS deactivation maps) (D). $p<$ 0.005, 20-voxel threshold extent.

regions and rolandic opercula bilaterally and the insular cortex, inferior parietal lobule, supramarginal gyrus, superior temporal gyrus, and rolandic operculum on the right in the TPM group (figure 2D, figure e-2). Group differences on the right were due mainly to impaired deactivation compared to LEV and ZNS (masked inclusively for LEV and ZNS group deactivation maps; shown in blue in figure $2 \mathrm{D}$ ). Left-sided changes were located within LEV group activation maps and hence were due to greater task-relevant activation in TPM (shown in red in figure 2D). 
There were no regions of greater activation in ZNS-treated patients compared to those on TPM.

Table 3 gives a more detailed anatomic description of resultant regions from the individual group comparisons.

DISCUSSION In this study, we demonstrate language paradigm-specific fMRI effects with a decrease in frontal lobe and parietal lobe activations common to ZNS and TPM and impaired deactivation in tasknegative networks seen only for TPM.

Our results concur with findings from previous studies on TPM reporting decreased task-relevant frontal activation or impaired deactivation of task-negative networks ${ }^{4,5,7,8}$ and demonstrate both mechanisms in

Table 3 Anatomic description and peak activations of resultant areas from group comparisons

Region

Patients on LEV > patients on TPM

Left dorsal parietal

Left middle frontal gyrus

Left inferior temporal gyrus

Left superior temporal gyrus

Left supplementary motor area

Patients on LEV > patients on ZNS

Left inferior frontal gyrus

Left dorsal parietal

Left inferior temporal gyrus

Left middle frontal gyrus

Right inferior frontal gyrus

Right middle frontal gyrus

Patients on TPM > patients on LEV

Left rolandic operculum

Left temporal lobe

Left superior temporal gyrus

Right rolandic operculum/inferior parietal lobule/supramarginal gyrus

Right temporal lobe

Right superior temporal gyrus

Right precuneus

Patients on TPM > patients on ZNS

Left inferior frontal gyrus

Left insula

Left anterior cingulate gyrus

Right rolandic operculum/inferior parieta lobule/supramarginal gyrus

Right insula/right rolandic operculum/right superior temporal gyrus

Right medial frontal gyrus
MNI coordinates $(x, y, z) \quad z$ Score $p$ Value
$-48,-43,43$

$-39,5,34$

$-54,-40,-20$

$-45,14,-11$

$-9,23,49$

$-42,5,34$

$-27,-64,40$

$-48,-46,-11$

$-45,32,28$

$48,32,-2$

$36,50,7$

$-36,-31,19$

$-42,-25,-11$

$-54,-7,4$

$45,-25,22$

$42,-19,-8$

$51,-13,7$

$9,-49,55$

$-48,-1,19$

$-30,-4,13$

$-18,35,16$

$54,-13,22$

$48,-7,10$

$12,56,16$
3.94

3.90

3.55

3.38

2.90

4.07

3.93

3.35

3.68

3.51

3.20

3.48

3.14

3.17

3.88

3.36

3.16

2.96

0.002

$4.07<0.001$

$3.35<0.001$

$3.98<0.001$

$3.70<0.001$

$3.31<0.001$
$3.00 \quad 0.001$

Abbreviations: LEV = levetiracetam, MNI = Montreal Neurological Institute, TPM = topiramate, ZNS = zonisamide.

Coordinates are given in MNI space. a larger group of patients. We found that ZNS is associated with similar dysfunctional networks of task activation but that impaired deactivation appears to be specific to TPM, as shown in comparison to both LEV- and ZNS-treated patients.

The verbal fluency fMRI task usually leads to activation of frontal lobe areas, including most consistently the dominant IFG, MFG, anterior cingulate, and precentral cortices, as well as the insular, superior temporal, and parietal cortices and the cerebellum (contralateral to frontal activation). ${ }^{14-16}$ Dorsolateral frontal and parietal cortices activated by the verbal fluency task are also implicated in functional networks of working memory and attention. ${ }^{17,18}$ Given the low-demand control condition in the verbal fluency task used in this study, activation of cognitive networks supporting general task performance, i.e., the working memory and sustained attention system, becomes apparent with increasing task demand, in addition to activation of language-specific frontal networks. ${ }^{19}$ Hence, decreased activation in both regions relevant for expressive language function (i.e., IFG, MFG) and general cognitive task performance, i.e., the parietal cortex for sustained attention and the frontoparietal working memory network, suggests suppression of several higher-level cognitive domains by TPM and ZNS.

In TPM-treated patients, fMRI changes involved both activation and deactivation networks. Taskrelated deactivation refers to a decrease in blood oxygen level-dependent signal during demanding motor or cognitive tasks compared to less demanding states such as resting. Deactivation likely occurs because neural processes during these less demanding states are interrupted by engagement with the task and a shift from internal to external information processing. Successful task execution has been associated with effective deactivation of task-negative areas. ${ }^{20,21}$ It has recently been demonstrated that the DMN is segregated into subspecialized nodes and, more relevant to our study, that there is functional heterogeneity across DMN nodes with respect to different language tasks. ${ }^{21,22}$ In addition, areas involved in semantic and language processing show a large overlap with regions of the DMN (i.e., angular gyrus, medial prefrontal cortex, IFG, posterior cingulate, ventral temporal lobe). ${ }^{19}$ Deactivation in those overlapping regions can be modulated by both task content, i.e., high semantic demands reducing deactivation, and increased task demands or difficulty leading to increased deactivation. ${ }^{19,21}$ In our study, patients on TPM failed to deactivate task-relevant DMN nodes compared to patients taking ZNS or LEV, associated with more cognitive impairment than those on ZNS, and these include areas implicated in language processing (i.e., right inferior parietal lobule, supramarginal gyrus). In addition, direct comparison 
to ZNS reveals that TPM leads to failed deactivation of language-task relevant DMN nodes on the right but increased activation of language-relevant task-positive regions on the left (figure 2D, figure e-2). The latter, as demonstrated by psychometric out-of-scanner data, is ineffective.

A particular strength of our study is the big sample size. As a limitation, the statistical threshold used for the second-level analysis, i.e., $p<0.005$ uncorrected, 20 -voxel threshold extent, enables an exploratory view of the differences between AED treatment groups, although peak activations within implicated regions almost all survive $p<0.001$ uncorrected (table 3). Findings need to be confirmed in a follow-up study with larger patient groups. Interpretation of findings may be limited in that patients on TPM and ZNS were compared only to those on LEV. However, the reported effects of LEV ${ }^{10}$ have been toward restoration of normal activation patterns, justifying our choice as a patient control group. Detrimental effects of TPM were demonstrated even when compared to ZNS only. In addition, LEV is comparable to ZNS and TPM in its clinical application of a commonly used broad-spectrum AED.

There is a potential case selection bias because our study included only patients who continued treatment on TPM and ZNS and hence may have benefitted more and experienced fewer side effects than those who stopped these medications. A further potential confounder is the reason why a particular medication was chosen for a patient. All 3 drugs are broad-spectrum AEDs with an uncomplicated interaction profile with other AEDs and have been established for several years in the treatment of epilepsy in general and in polytherapy in refractory epilepsy. ${ }^{23-25}$ However, we cannot control for treatment preferences in certain patients, e.g., choosing TPM in those who also have migraines. More than 10 consultants are involved in epilepsy treatment at our center, supporting that the AED profile of our groups is not driven by the personal choices of a few individuals; however, AED choices in our epilepsy center may differ from those in others.

The majority of patients were on comedication, which may have contributed to poor cognitive performance and contributed noise to the data. It has been shown that every additional AED leads to further cognitive impairment. ${ }^{26}$ AED plasma concentrations were not known at the time of scanning. Although we cannot fully control for effect of comedication, we matched groups for the median number of AEDs, and individual comedication AEDs were included as a regressor of no interest in the fMRI analysis model, which is a standard methodology in fMRI analysis. Although future studies in patients on monotherapy are necessary to fully control for comedication effects, we stress that considering which AED to choose next in a treatment-refractory patient already on polytherapy is a common clinical dilemma, and findings here may eventually help the clinician's choice.

Out-of-scanner psychometric data were available in only a subset of patients.

Because of the retrospective study design, the effect of seizures on our findings could not be quantified in terms of frequency, severity, or proximity to scan time. All patients had refractory epilepsy; we therefore assume similar effects of seizures in all groups.

Although all patients had focal epilepsy, different epilepsy syndromes were included (table e-1). Our study characterizes TPM/ZNS-related disruption of language and working memory networks in a relatively large group of presurgical candidates of a big tertiary epilepsy referral center. Although our findings are not fully generalizable because medical treatment strategies and drug choices may differ across epilepsy centers and countries, observed fMRI results in this study still provide valuable information for interpreting clinical language fMRI scans in a variety of patients.

With respect to clinical applications, task-, region-, and AED-specific effects of TPM and ZNS may help to identify patients at risk of developing AED-related side effects at an early stage of treatment. So far, group studies have shown high sensitivity of pharmacofMRI, detecting negative drug effects on neuronal networks even after a single-dose application. ${ }^{7}$ Prospective longitudinal studies, enabling fMRI and psychometric data collection before and when TPM and ZNS are initiated (ideally with plasma levels), will help to elucidate why certain patients develop cognitive side effects on a specific drug and others do not. To date, pharmaco-fMRI could not be implemented as a standard tool at single-patient level because of the costs and because the limits of normal and abnormal activations at single-patient level so far cannot be quantified.

Identifying language lateralization with fMRI is crucial for risk assessment during planning for epilepsy surgery. ${ }^{27}$ It would thus be important to establish whether fMRI changes due to TPM and ZNS can lead to mislateralization of language. In this study, because groups were matched for laterality index to increase the yield by including patients regardless of language lateralization, we cannot comment on a potential effect of TPM and ZNS on laterality indexes, and this will be more appropriately answered in longitudinal studies before and after treatment initiation.

\section{AUTHOR CONTRIBUTIONS}

Dr. Wandschneider: drafting/revising the manuscript, analysis and interpretation of data, statistical analysis. J. Burdett: drafting/revising the manuscript, data collection. L. Townsend: drafting/revising the manuscript, data collection. A. Hill: drafting/revising the manuscript, data collection. Dr. Thompson: drafting/revising the manuscript, study concept or 
design, interpretation of data, study supervision. Dr. Duncan: drafting/ revising the manuscript, study concept, interpretation of data, study supervision. Dr. Koepp: drafting/revising the manuscript, study concept or design, interpretation of data, study supervision.

\section{STUDY FUNDING}

We are grateful to the Wolfson Trust and the Epilepsy Society for supporting the Epilepsy Society MRI scanner. This work was undertaken at University College London Hospitals/University College London, which received a proportion of funding from the Department of Health's National Institute for Health Research University College London Hospitals Biomedical Research Centre funding scheme.

\section{DISCLOSURE}

B. Wandschneider, J. Burdett, L. Townsend, A. Hill, and P. Thompson report no disclosures relevant to the manuscript. J.S. Duncan serves on the scientific advisory boards for and/or has received funding for travel from GE Healthcare, GSK, Eisai, and UCB Pharma. M.J. Koepp served on a scientific advisory board of GE Healthcare and has received honoraria for lectures from Eisai and UCB Pharma. Go to Neurology.org for full disclosures.

Received May 11, 2016. Accepted in final form January 3, 2017.

\section{REFERENCES}

1. Arif H, Buchsbaum R, Weintraub D, Pierro J, Resor SR, Hirsch LJ. Patient-reported cognitive side effects of antiepileptic drugs: predictors and comparison of all commonly used antiepileptic drugs. Epilepsy Behav 2009;14: 202-209.

2. Mula M, Trimble MR. Antiepileptic drug-induced cognitive adverse effects: potential mechanisms and contributing factors. CNS Drugs 2009;23:121-137.

3. Ojemann LM, Ojemann GA, Dodrill CB, Crawford CA, Holmes MD, Dudley DL. Language disturbances as side effects of topiramate and zonisamide therapy. Epilepsy Behav 2001;2:579-584.

4. Jansen JFA, Aldenkamp AP, Marian Majoie HJ, et al. Functional MRI reveals declined prefrontal cortex activation in patients with epilepsy on topiramate therapy. Epilepsy Behav 2006;9:181-185.

5. Szaflarski JP, Allendorfer JB. Topiramate and its effect on fMRI of language in patients with right or left temporal lobe epilepsy. Epilepsy Behav 2012;24:74-80.

6. De Ciantis A, Muti M, Piccolini C, et al. A functional MRI study of language disturbances in subjects with migraine headache during treatment with topiramate. Neurol Sci 2008;29(suppl 1):S141-S143.

7. Yasuda CL, Centeno M, Vollmar C, et al. The effect of topiramate on cognitive fMRI. Epilepsy Res 2013;105: 250-255.

8. Tang Y, Xia W, Yu X, et al. Altered cerebral activity associated with topiramate and its withdrawal in patients with epilepsy with language impairment: an fMRI study using the verb generation task. Epilepsy Behav 2016;59: 98-104.

9. Kwon O-Y, Park S-P. Zonisamide decreases currentsource density of high beta frequency of electroencephalogram. J Epilepsy Res 2013;3:63-69.
10. Wandschneider B, Stretton J, Sidhu M, et al. Levetiracetam reduces abnormal network activations in temporal lobe epilepsy. Neurology 2014;83:1508-1512.

11. Lieberman MD, Cunningham WA. Type I, type II error concerns in fMRI research: re-balancing the scale. Soc Cogn Affect Neurosci 2009;4:423-428.

12. Wilke M, Lidzba K. LI-tool: a new toolbox to assess lateralization in functional MR-data. J Neurosci Methods 2007;163:128-136

13. Baxendale $S$, Thompson PJ, Duncan JS. Improvements in memory function following anterior temporal lobe resection for epilepsy. Neurology 2008;71:1319-1325.

14. Friedman L, Kenny JT, Wise AL, et al. Brain activation during silent word generation evaluated with functional MRI. Brain Lang 1998;64:231-256.

15. Phelps EA, Hyder F, Blamire AM, Shulman RG. FMRI of the prefrontal cortex during overt verbal fluency. Neuroreport 1997;8:561-565.

16. Woermann FG, Jokeit $H$, Luerding $R$, et al. Language lateralization by Wada test and fMRI in 100 patients with epilepsy. Neurology 2003;61:699-701.

17. Owen AM, McMillan KM, Laird AR, Bullmore E. Nback working memory paradigm: a meta-analysis of normative functional neuroimaging studies. Hum Brain Mapp 2005;25:46-59.

18. Coull JT. Neural correlates of attention and arousal: insights from electrophysiology, functional neuroimaging and psychopharmacology. Prog Neurobiol 1998;55:343-361.

19. Binder JR, Desai RH, Graves WW, Conant LL. Where is the semantic system? A critical review and meta-analysis of 120 functional neuroimaging studies. Cereb Cortex 2009; 19:2767-2796.

20. Raichle ME, MacLeod AM, Snyder AZ, Powers WJ, Gusnard DA, Shulman GL. A default mode of brain function. Proc Natl Acad Sci USA 2001;98:676-682.

21. Seghier ML, Price CJ. Functional heterogeneity within the default network during semantic processing and speech production. Front Psychol 2012;3:281.

22. Andrews-Hanna JR. The brain's default network and its adaptive role in internal mentation. Neuroscientist 2012; 18:251-270.

23. Pulman J, Jette N, Dykeman J, Hemming K, Hutton JL, Marson AG. Topiramate add-on for drug-resistant partial epilepsy. Cochrane Database Syst Rev 2014:CD001417.

24. Carmichael K, Pulman J, Lakhan SE, Parikh P, Marson AG. Zonisamide add-on for drug-resistant partial epilepsy. Cochrane Database Syst Rev 2013:CD001416.

25. Mbizvo GK, Dixon P, Hutton JL, Marson AG. Levetiracetam add-on for drug-resistant focal epilepsy: an updated Cochrane review. Cochrane Database Syst Rev 2012: CD001901.

26. Witt J-A, Elger CE, Helmstaedter C. Adverse cognitive effects of antiepileptic pharmacotherapy: each additional drug matters. Eur Neuropsychopharmacol 2015;25: 1954-1959.

27. Duncan JS, Winston GP, Koepp MJ, Ourselin S. Brain imaging in the assessment for epilepsy surgery. Lancet Neurol 2016;15:420-433. 


\title{
Neurology
}

\author{
Effect of topiramate and zonisamide on fMRI cognitive networks \\ Britta Wandschneider, Jane Burdett, Lucy Townsend, et al. \\ Neurology 2017;88;1165-1171 Published Online before print February 17, 2017 \\ DOI 10.1212/WNL.0000000000003736
}

This information is current as of February 17, 2017

$\begin{array}{ll}\begin{array}{l}\text { Updated Information \& } \\ \text { Services }\end{array} & \begin{array}{l}\text { including high resolution figures, can be found at: } \\ \text { http://n.neurology.org/content/88/12/1165.full }\end{array} \\ \text { Supplementary Material } & \text { Supplementary material can be found at: } \\ \text { http://n.neurology.org/content/suppl/2017/02/17/WNL.0000000000003 } & 736 . D C 1 \\ & \text { This article cites } 24 \text { articles, } 3 \text { of which you can access for free at: } \\ & \text { http://n.neurology.org/content/88/12/1165.full\#ref-list-1 } \\ \text { References } & \text { This article has been cited by } 3 \text { HighWire-hosted articles: } \\ & \text { http://n.neurology.org/content/88/12/1165.full\#\#otherarticles } \\ \text { Citations } & \text { This article, along with others on similar topics, appears in the } \\ & \text { following collection(s): } \\ \text { All Epilepsy/Seizures } \\ \text { http://n.neurology.org/cgi/collection/all_epilepsy_seizures } \\ \text { All Neuropsychology/Behavior } \\ \text { http://n.neurology.org/cgi/collection/all_neuropsychology_behavior } \\ \text { Antiepileptic drugs } \\ \text { http://n.neurology.org/cgi/collection/antiepileptic_drugs } \\ \text { Functional neuroimaging } \\ \text { http://n.neurology.org/cgi/collection/functional_neuroimaging } \\ \text { Information about reproducing this article in parts (figures,tables) or in } \\ \text { its entirety can be found online at: } \\ \text { http://www.neurology.org/about/about_the_journal\#permissions } \\ \text { Information about ordering reprints can be found online: } \\ \text { http://n.neurology.org/subscribers/advertise }\end{array}$

Neurology ${ }^{\circledR}$ is the official journal of the American Academy of Neurology. Published continuously since 1951, it is now a weekly with 48 issues per year. Copyright Copyright ( 2017 The Author(s). Published by Wolters Kluwer Health, Inc. on behalf of the American Academy of Neurology. All rights reserved. Print ISSN: 0028-3878. Online ISSN: 1526-632X.

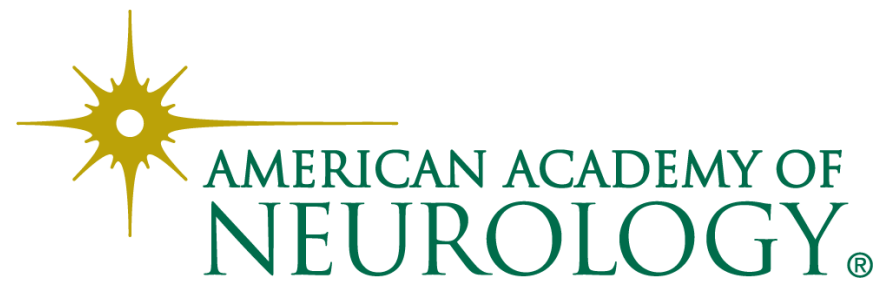

